\title{
Experimental Verification of Reversed Cherenkov Radiation in Left-Handed Metamaterial
}

\author{
Sheng Xi ${ }^{1,2}$ Hongsheng Chen, ${ }^{1,2, *}$ Tao Jiang, ${ }^{1}$ Lixin Ran, ${ }^{1, \dagger}$ Jiangtao Huangfu, ${ }^{1}$ Bae-Ian Wu,,${ }^{1,2}$ \\ Jin Au Kong, ${ }^{1,2}$ and Min Chen ${ }^{3, *}$ \\ ${ }^{1}$ The Electromagnetics Academy at Zhejiang University, Zhejiang University, Hangzhou 310027, China \\ ${ }^{2}$ Research Laboratory of Electronics, Massachusetts Institute of Technology, Cambridge, Massachusetts 02139, USA \\ ${ }^{3}$ Department of Physics, Massachusetts Institute of Technology, Cambridge, Massachusetts 02139, USA
}

(Received 22 July 2009; published 2 November 2009)

By using a phased electromagnetic dipole array to model a moving charged particle, we experimentally verified a reversed Cherenkov radiation in the left-handed media in the frequency range from 8.1 to 9.5 GHz. Our results demonstrate the feasibility of new types of particle detectors and radiation generators.

DOI: 10.1103/PhysRevLett.103.194801

PACS numbers: $41.60 . \mathrm{Bq}, 41.20 . \mathrm{Jb}$

In 1934, Cherenkov discovered experimentally the coherent radiation when some media were bombarded by fast-moving electron beams [1]. Frank and Tamm established the theory of Cherenkov radiation (CR) in the framework of classical electrodynamics in 1937 [2]. The theory shows that CR will radiate only when the speed of the charge is larger than the speed of light in the medium. Since then, CR has been widely applied in high-energy physics. One of the important applications is Cherenkov counters, which are used to determine various properties of a charged particle such as its velocity, charge, direction of motion, and energy. These properties are important in the identification of different particles. The discovery of antiproton [3] is a good example of the application of CR, as well as the discovery of the $J$ particle [4], in which Chen designed the six large Cherenkov counters.

In conventional media, the radiation angle of $\mathrm{CR} \theta_{c}$, which is the angle between the direction of wave propagation and charge velocity, is always between $0^{\circ}$ and $90^{\circ}$. Thus, the charged particle will interfere with the detection of the forward radiated waves. In 1968, Veselago predicted the abnormal reversed Cherenkov radiation in the lefthanded metamaterials (LHMs) with simultaneously negative permittivity and permeability [5], allowing the backward emitted wave to be easily separable from the emitting particles. More theoretical frameworks have been proposed since then [6]. The first experimental attempt using a beam of charged particles with metamaterials was carried out in a waveguide [7]. Although the result cannot be decisively concluded as a backward CR, the observation of the transmission peak in the left-handed band is an indirect observation of the wakefield generation [7]. In short, experimental verification of backward CR is still very rare. The lack of LHMs for transverse magnetic (TM) polarized waves has been one of the main obstacles. Because of the rotational symmetry of the cylindrical configuration for $\mathrm{CR}$, the experimental verification of the reversed CR requires metamaterials for transversely magnetic polarized waves, which requires negative permeability along the $\phi$ direction and negative permittivities along the $\rho$ and $z$ directions. Since Pendry et al. first showed that the metallic rod arrays [8] and the split-ring resonators (SRRs) [9] exhibited negative permittivity and negative permeability, respectively, most of the LHMs realized so far have been for transverse electric (TE) polarized waves [10], which is not suitable for backward CR detection. The second reason is that the radiation power of CR increases with its frequency; therefore, the optical spectrum is more fitted for detection, but LHMs are more commonly used in the microwave frequency band due to the restraint of the present technique of fabrication in the high frequency band as well as the loss increment in the metal as it scales to optical spectrum. These increase the difficulty of the experimental verification of the reversed CR effect.

In this Letter, a new type of TM-LHM is experimentally fabricated for $\mathrm{CR}$ detection. In order to increase the radiation power, a waveguide with an array of open slots is designed to model a moving charged particle, which enables the verification of reversed $\mathrm{CR}$ in the microwave band. The equivalence between the dipole array and the moving charged particle is theoretically proven. Backward radiation is observed in the left-handed band with the slot waveguide surrounded with the LHMs, successfully demonstrating the reversed CR effect in LHMs.

The configuration and dimensions of the sample are shown in Fig. 1. A single layer of the whole structure is copper strips with thickness $t_{m}=17.5 \mu \mathrm{m}$ printed on both sides of a thin sheet of polytetrafluoroethylene substrate with thickness $t=33 \mu \mathrm{m}$ and relative permittivity 2.1 , as shown by the photo on the right-hand side of Fig. 1. The periodicity of the unit in the $x-z$ plane is $a=3 \mathrm{~mm}$. The width of the margin of a unit cell is $b=0.271 \mathrm{~mm}$. The dimensions of the conducting rods and the split-ring structures are $d_{r}=0.249 \mathrm{~mm}, d_{g}=0.227 \mathrm{~mm}$, and $w=$ $0.317 \mathrm{~mm}$. The orthogonal copper rods provide negative permittivity in the $x$ - $z$ plane. The two $L$-shaped metal strips on the top side couple with the two on the bottom side to form a magnetic resonator, providing a negative perme- 


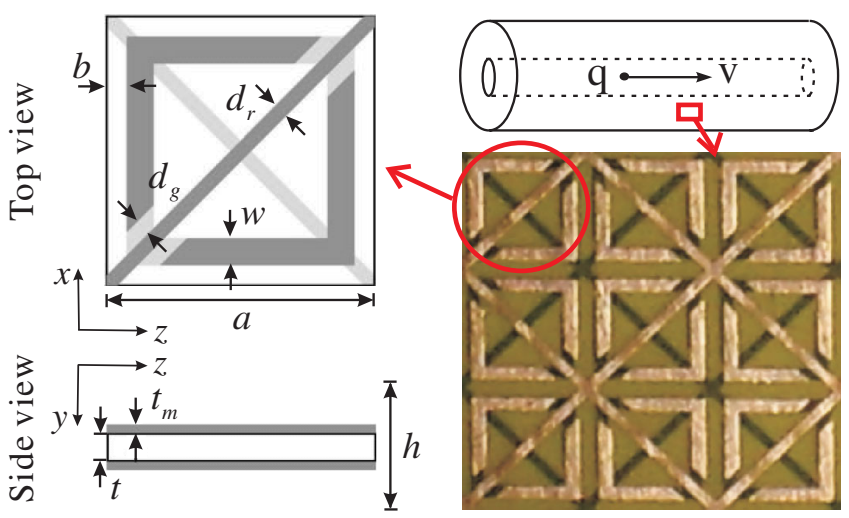

FIG. 1 (color online). Configuration of the TM-LHM for experiment. In the top view, the dark (light) gray trips represent the copper printed on the top (bottom) of the substrate.

ability for the $y$-polarized magnetic fields. The single layer is repeated along the $y$ axis with period $h$, sandwiched by soft plastic foams, whose electromagnetic properties are close to those of the free space, forming a block of material.

A prism shaped sample shown in the inset of Fig. 2(a) is fabricated. The angle is $\tau=18.4^{\circ}$ and there are 23 cells on the edge of the prism along the $x$ axis. The sample is measured in the microwave anechoic chamber. The farfield pattern when a TM wave incidents onto the prism is measured. An Agilent 8722ES network analyzer connecting the transmitter and the receiver antennas to its two ports is used to measure the far-field power. The half power bandwidth of the transmitter antenna is $19.5^{\circ}$ at $8.5 \mathrm{GHz}$. The refractive index extracted from the measured results is shown in Fig. 2(a). The negative refractive index region is
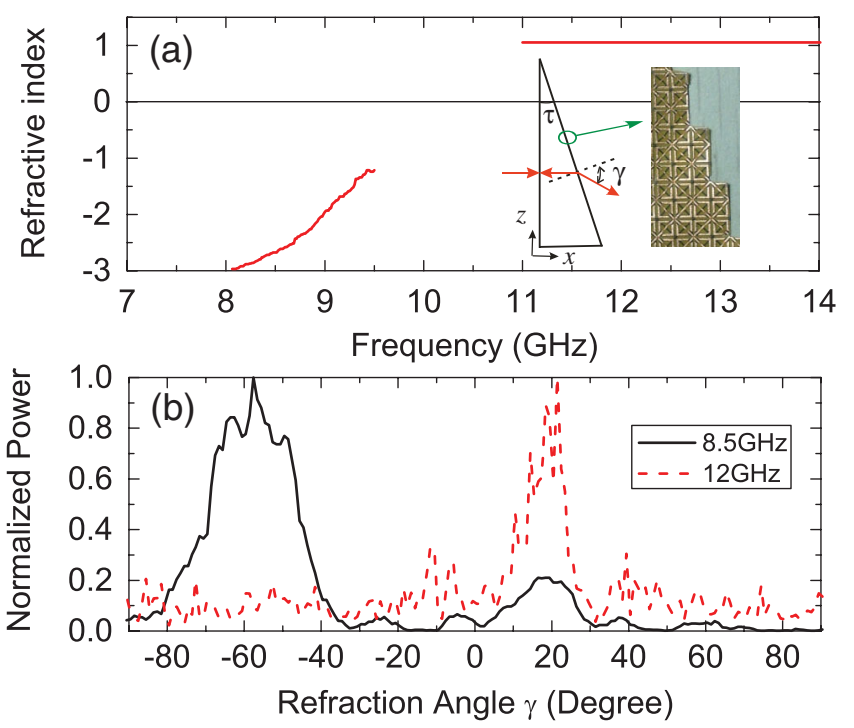

FIG. 2 (color online). (a) The refractive index of the TM-LHM calculated from the measured results. The periodicity along the $y$ axis is $h=1.64 \mathrm{~mm}$. (b) The normalized far-field pattern of the prism experiment at 8.5 and $12 \mathrm{GHz}$, respectively. from 8.1 to $9.5 \mathrm{GHz}$. In the frequency band from 11 to $14 \mathrm{GHz}$, most of the power is positively refracted. The measured power as a function of the refraction angle at $8.5 \mathrm{GHz}$ is plotted in Fig. 2(b), where most of power is refracted to the negative region. The refraction angle of the maximum power at $8.5 \mathrm{GHz}$ is $58^{\circ}$, corresponding to a refractive index of -2.7 , while at $12 \mathrm{GHz}$, the refraction angle is about $21^{\circ}$, corresponding to a refractive index of 1.1 .

The radiated power of a fast-moving charged particle at microwave frequency is very small. In order to improve the radiation power, we use a phased dipole array to model the charged particle. Here we show that such modeling is reasonable. When a charged particle with charge $q$ moves along the $z$ axis with velocity $v$, as shown by Fig. 3(a), the current density of the moving charge is [11]

$$
\bar{J}_{p}(\bar{r}, t)=\hat{z} q v \frac{\delta(\rho)}{2 \pi \rho} \delta(z-v t)
$$

where $\hat{z}$ is the unit vector along $z$ axis, $\rho$ represents the radial dimension in the $x-y$ plane, $\delta$ is the Dirac delta function, and $t$ is the time. This current is not time harmonic. Through the use of Fourier transform, we obtain the expression of this current in frequency domain as

$$
\bar{J}_{p}(\bar{r}, \omega)=\hat{z} \frac{q}{4 \pi^{2} \rho} \delta(\rho) e^{i k_{z} z},
$$

where $\omega$ is the frequency, $k_{z}=\omega / v$ is the wave vector along the $z$ axis, $e$ is the exponential function, and $i$ is the unit of imaginary numbers. Consider a phased dipole array of an infinite number of elements pointing in the $\hat{z}$ direction and placed along the $z$ axis with periodicity $\Delta$, as shown by Fig. 3(b). Each dipole has a current with amplitude $I$, an infinitesimal length $l$, and a phase shift $\alpha$ relative to its adjacent element. Assuming the dipole array works at the frequency $\omega_{0}$, the current density of the $n$th dipole is $\bar{J}_{n}(\bar{r}, t)=\hat{z} I l \delta(\rho) /(2 \pi \rho) e^{i n \alpha-i \omega_{0} t}$. The current density of the dipole array is the sum of the current densities of all the dipoles:

$$
\bar{J}_{d}(\bar{r}, t)=\hat{z} \frac{I l \delta(\rho)}{2 \pi \rho} \sum_{n=-\infty}^{\infty} \delta(z-n \Delta) e^{i n \alpha-i \omega_{0} t} .
$$

Also through the Fourier transform, we get the current density of the dipole array in the frequency domain:

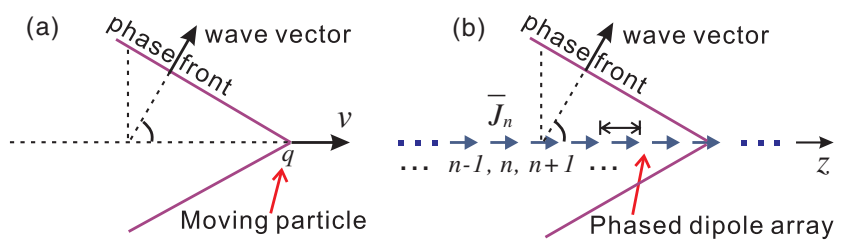

FIG. 3 (color online). The radiation of (a) a moving charged particle and (b) a phased dipole array composed of an infinite number of dipoles along the $z$ axis. 


$$
\bar{J}_{d}(\bar{r}, \omega)=\hat{z} \frac{I l \delta(\rho)}{2 \pi \rho} \delta\left(\omega-\omega_{0}\right) \sum_{n=-\infty}^{\infty} \delta(z-n \Delta) e^{i n \alpha} .
$$

Assuming $\alpha=k_{z} \Delta$ and forcing $\Delta=l$ so that the adjacent dipoles connect each other, Eq. (4) then becomes

$$
\begin{aligned}
\bar{J}_{d}(\bar{r}, \omega) & =\hat{z} \frac{I \delta(\rho)}{2 \pi \rho} \delta\left(\omega-\omega_{0}\right) \int_{-\infty}^{\infty} \delta(z-n l) e^{i k_{z} n l} d(n l) \\
& =\hat{z} \frac{I \delta\left(\omega-\omega_{0}\right)}{2 \pi \rho} \delta(\rho) e^{i k_{z} z}
\end{aligned}
$$

It is obvious to see that the current density of a moving charged particle as shown by Eq. (2) and the current density of the phased dipole array as shown by Eq. (5) are very similar except that the moving particle contains the whole spectrum of the frequencies and the dipole array has only one working frequency $\omega_{0}$. However, at the single frequency $\omega_{0}$, the behavior of the moving charged particle and the phased dipole array will be exactly the same. Thus, by setting up the proposed array with $\alpha=k_{z} \Delta$ and $\Delta=l$, the radiation properties of the frequency component $\omega_{0}$ radiated by the moving particle with velocity $v=\omega_{0} / k_{z}$ can be verified.

Next, we designed a slot waveguide to imitate the phased dipole array. The configuration of the slot waveguide is shown by the inset of Fig. 4. If the dimensions of the slots $d_{1}$ and $d_{2}$ are relatively small compared with the wavelength, and $\Delta=d_{1}$, with the additional assumption that there are an infinite number of slots in total, then we can get $k_{z}$ to be a constant for each frequency in the basic mode $\mathrm{TE}_{01}$. The magnitudes of the magnetic fields in all of the slots can also be treated as a constant for each frequency. Setting the magnitude of the magnetic fields in each slot as $\hat{y} H_{0}$, then the equivalent surface current of the $n$th slot is

$$
\bar{J}_{s}(\bar{r}, t)=\hat{z} H_{0} e^{i(n-1) \alpha-i \omega_{0} t},
$$

with $\alpha=k_{z} \Delta$ and $k_{z}=\omega_{0} / v$. Thus, the slot waveguide

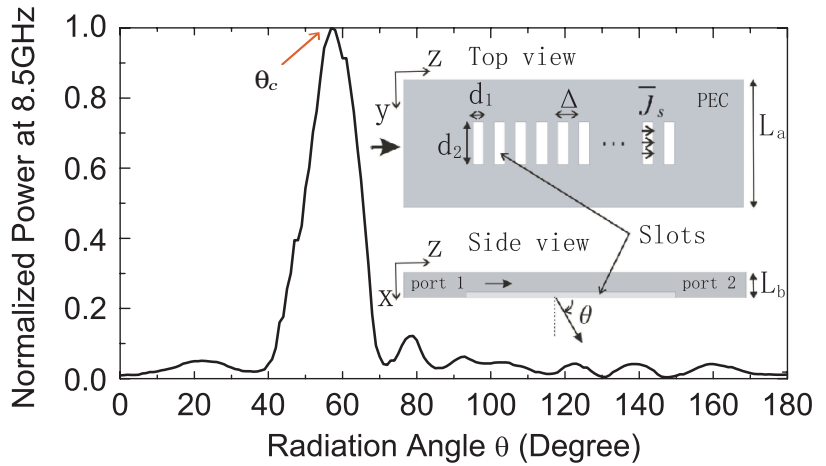

FIG. 4 (color online). The normalized radiation pattern of the slot waveguide at $8.5 \mathrm{GHz}$. The inset shows a moving particle with radiation angle $\theta_{c}=58^{\circ}$. can be considered as a phased dipole array. However, when $d_{1}$ and $d_{2}$ are too small, the radiated power will be too small to detect. In order to increase the radiation efficiency while maintaining the mode in the waveguide, the slot waveguide with dimensions $d_{1}=5 \mathrm{~mm}, d_{2}=12 \mathrm{~mm}$, $\Delta=10 \mathrm{~mm}, L_{a}=20 \mathrm{~mm}$, and $L_{b}=10 \mathrm{~mm}$ is fabricated. There are 14 slots in total along $z$ axis. The source is plugged into port 1 and port 2 is connected with an impedance matcher. The far-field pattern of the wave radiated by the slot waveguide is measured in a microwave anechoic chamber. At $8.5 \mathrm{GHz}$, the pattern is shown in Fig. 4. The radiation power is mainly concentrated in the forward region and the angle of the main lobe is $\theta_{c}=58^{\circ}$. Equivalently, this corresponds to a charged particle moving at $v=1.9 c / n$, where $c / n$ is the phase velocity of light. For the other frequencies, the pattern is similar to Fig. 4; however, the angle of the main lobe will decrease as the frequency increases.

In the verification of reversed CR in LHMs, the far field of the radiated waves needs to be measured. However, it is not practical to build a large block of LHM and measure the far field inside of the metamaterial. Thus, we proceed to measure the far field of the radiation passing through a small block of LHM. If the moving charged particle is surrounded by a slab of LHMs, the detected wave at the far field is still forward, no matter if the slab is LHM or RHM, as shown by Fig. 5(a), where the solid line and the dotted line indicate the radiation behavior in LHM and RHM, respectively. Therefore, in order to decisively determine the radiation properties inside of the metamaterial, a prism of the LHM sample is used for the experimental verification. From the schematic of Fig. 5(b), we see that if CR in the sample is forward, then the angle $\gamma$ should always be positive, as indicated by the dotted line. Whenever negative $\gamma$ is observed, CR in the LHM sample must be reversed, as indicated by the solid line in Fig. 5(b). Since we are measuring the radiation pattern on the $\phi=$ const plane, it is not necessary to construct a conical sample to wrap the slot waveguide. Instead, a planar prism of the LHM put close to the slot waveguide, as shown by the inset of Fig. 5(c), can be used for demonstration. However, it should be noted that although we only measured the radiation pattern on the $\phi=$ const plane, the measured far-field pattern and the radiation field inside of the LHMs are rotationally symmetric along the $z$ direction due to the rotational symmetric configuration of the $\mathrm{CR}$ setup. The measured far-field pattern at $8.5 \mathrm{GHz}$ is shown by Fig. 5(c). This is equivalent to the frequency component at $8.5 \mathrm{GHz}$ radiated by the charged particle moving inside of the LHM prism. From the result, we see that most of the radiation power is in the negative region and the refracted angle at far field is $-22^{\circ}$. Our previous prism experiment shows that the refractive index at $8.5 \mathrm{GHz}$ is $n_{l}=-2.7$; therefore, we can calculate that the incident angle at the prism interface is $\theta_{i}=8.0^{\circ}$. Since the incident beam at the prism 

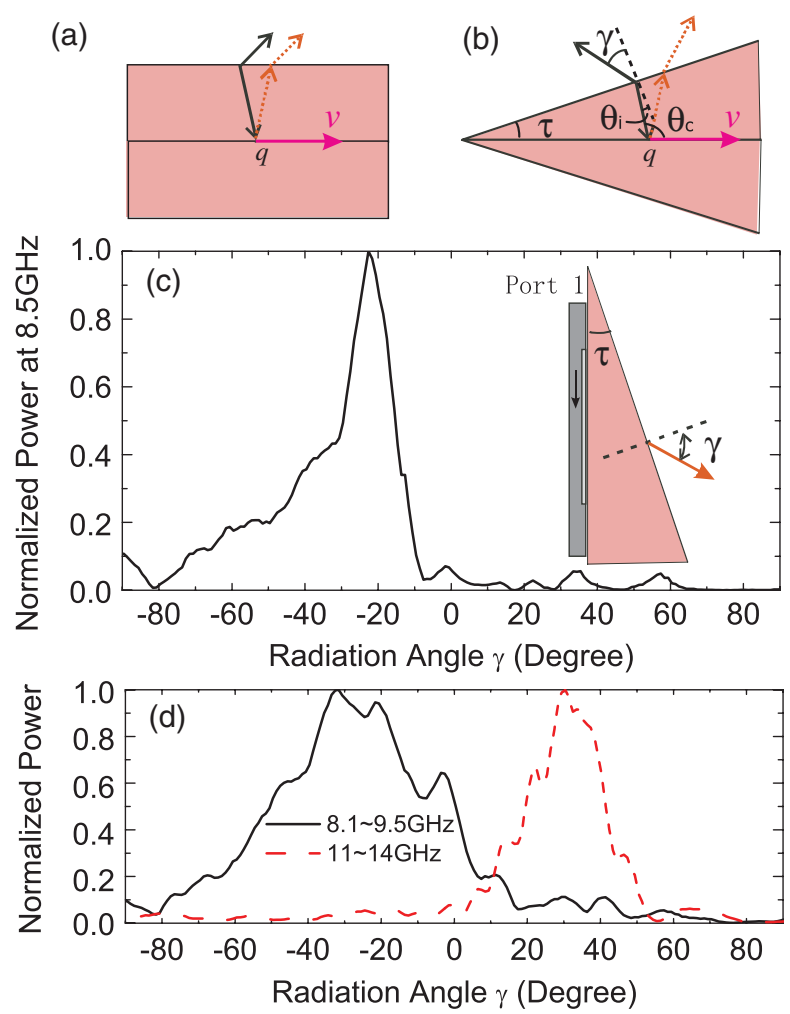

FIG. 5 (color online). (a) Radiation passing through a slab of LHM (solid line) and RHM (dotted line). (b) Radiation passing through a prism of LHM (solid line) and RHM (dotted line). (c) Reversed CR is observed at $8.5 \mathrm{GHz}$. The inset illustrates the experimental setup. The source is plugged in port 1. (d) Sum of the radiation power in each angle in the negative band (solid line) and positive band (dashed line).

interface originated from the CR radiation of the charged beam, the $\mathrm{CR}$ radiation angle is therefore calculated as $\theta_{c}=90^{\circ}-\theta_{i}+\tau=100.4^{\circ}$. From another viewpoint, the CR angle in the LHM can also be calculated directly from the CR condition [11], from where we can get $\theta_{c}=$ $\arccos \left(\frac{1}{n_{l} v / c}\right)=101.2^{\circ}$, which is in close agreement with the calculated result from the Snell's law at the prism interface. Thus, the radiation inside of the LHM is decisively concluded as a reversed CR. Figure 5(d) shows the radiation power in each angle. In the negative band from 8.1 to $9.5 \mathrm{GHz}$, most of the power is radiated in the backward directions, as shown by the solid line, while in the positive band from 11 to $14 \mathrm{GHz}$, the radiation power is mainly in the forward directions. These results are consistent with theoretical predictions.

In conclusion, a type of TM-LHM has been fabricated for verification of reversed CR. The negative refraction of this type of material is experimentally observed in the frequency band from 8.1 to $9.5 \mathrm{GHz}$. Phased dipole array is used to model the behavior of a moving charged particle in order to increase the radiation power. Backward CR is experimentally observed in the negative band of the material. The proposed type of material can be scaled to higher frequencies such as infrared and visible band. Since the radiated rays are in the backward direction with respect to the incident particle, the reverse $\mathrm{CR}$ will be valuable in the detection of charged particles.

This work is sponsored by the NNSFC (No. 60531020, No. 60801005, No. 60701007, No. 60990320, and No. 60990322), the FANEDD (No. 200950), the NCET07-0750, the ZJNSF (No. R1080320), the MEC (No. 200803351025), the ONR (No. N00014-06-1-0001), and the DAF (FA8721-05-C-0002).

\footnotetext{
*chenhs@ewt.mit.edu

†ranlx@zju.edu.cn

*chenm@mit.edu
}

[1] P. A. Cherenkov, Dokl. Akad. Nauk 2, 451 (1934) [Phys. Rev. 52, 378 (1937)].

[2] I. M. Frank and I.E. Tamm, Dokl. Akad. Nauk 14, 107 (1937) [Compt. Rend. (Dokl.) 14, 109 (1937)].

[3] O. Chamberlain, E. Segre, C. Wiegand, and T. Ypsilantis, Phys. Rev. 100, 947 (1955).

[4] J. J. Aubert, U. Becker, P. J. Biggs, J. Burger, M. Chen, G. Everhart, P. Goldhagen, J. Leong, T. McCorriston, T. G. Rhoades, M. Rohde, S. C. Ting, S. L. Wu, and Y. Y. Lee, Phys. Rev. Lett. 33, 1404 (1974).

[5] V. G. Veselago, Sov. Phys. Usp. 10, 509 (1968).

[6] C. Luo, M. Ibanescu, S. G. Johnson, and J.D. Joannopoulos, Science 299, 368 (2003); J. Lu, T. M. Grzegorczyk, Y. Zhang, J. Pacheco, B.-I. Wu, J. A. Kong, and M. Chen, Opt. Express 11, 723 (2003); Y. O. Averkov and V. M. Yakovenko, Phys. Rev. B 72, 205110 (2005); Y. P. Bliokh, S. Savel'ev, and F. Nori, Phys. Rev. Lett. 100, 244803 (2008); Z. Duan, B.-I. Wu, J. Lu, J. A. Kong, and M. Chen, J. Appl. Phys. 104, 063303 (2008).

[7] S. Antipov, L. Spentzouris, W. Gai, M. Conde, F. Franchini, R. Konecny, W. Liu, J. G. Power, Z. Yusof, and C. Jing, J. Appl. Phys. 104, 014901 (2008).

[8] J. B. Pendry, A. J. Holden, W. J. Stewart, and I. Youngs, Phys. Rev. Lett. 76, 4773 (1996).

[9] J. B. Pendry, A. J. Holden, D. J. Robbins, and W. J. Stewart, IEEE Trans. Microwave Theory Tech. 47, 2075 (1999).

[10] R. A. Shelby, D. R. Smith, and S. Schultz, Science 292, 77 (2001); H. J. Lezec, J. A. Dionne, and H. A. Atwater, Science 316, 430 (2007); J. Valentine, S. Zhang, T. Zentgraf, E. Ulin-Avila, D. A. Genov, G. Bartal, and X. Zhang, Nature (London) 455, 376 (2008).

[11] J.A. Kong, Electromagnetic Wave Theory (EMW Publishing, Cambridge, MA, 2005). 